\title{
Influence du jerk sur le comportement dynamique d'un robot cartésien 3 axes
}

\author{
Richard Béarée ${ }^{1}$, Pierre-Jean Barre ${ }^{1, a}$, Éric Dumetz ${ }^{2}$, François Ghestem ${ }^{3}$ \\ ET HENRI DAVID ${ }^{4}$ \\ 1 Équipe de Recherche Technologique « CEMODYNE» (ERT int 1022), Laboratoire d'Électrotechnique et d'Électronique \\ de Puissance de Lille (L2EP) ENSAM, 8 avenue Louis XIV, 59046 Lille Cedex, France \\ 2 ERT CEMODYNE - L2EP USTL-Polytech'Lille, cité scientifique, 59655 Villeneuve d'Ascq, France \\ 3 ERT CEMODYNE - L2EP ENSAM, 8 avenue Louis XIV, 59046 Lille Cedex, France \\ 4 SEPRO-ROBOTIQUE, Rue Bessemer, ZI Les Ajoncs, BP 65, 85002 La Roche sur Yon, France
}

Reçu le 14 février 2005, accepté le 13 avril 2005

\begin{abstract}
Résumé - Cet article traite de l'influence particulière du jerk sur le comportement dynamique d'un système de positionnement à grande cadence. Le robot cartésien 3 axes, utilisé comme support d'étude, est destiné au déchargement des presses à injecter le plastique. Dans ce type d'application, les critères de performances privilégiés sont la rapidité de mouvement et l'amplitude des oscillations liées aux modes de structure sollicités par la dynamique rapide du positionnement. Les performances d'une loi de mouvement à jerk contrôlé sont évaluées à partir d'un modèle analytique du système. On démontre que ce paramètre permet, d'une part, de maîtriser le comportement vibratoire des axes et, d'autre part, de conserver un temps de cycle acceptable en agissant sur les gains de boucle. Enfin, des essais expérimentaux viennent confirmer les résultats théoriques.
\end{abstract}

Mots clés : Jerk / robot cartésien / dynamique vibratoire / système de positionnement

\begin{abstract}
Jerk time influence on residual vibration and movement time of a 3-axis Cartesian robot. The focus of this paper is on the particular influence of the jerk limited profile on the dynamic behaviour of a 3-axis Cartesian robot. This robot is used for the unloading of the moulding machines. The predominating performances criteria are the movement time and the residual vibrations induced by high dynamical demands. The performances of a jerk limited movement law are evaluated using an analytical model of the controlled system. We show that the jerk time parameter can be used to limit the residual vibrations while preserving the movement time by a combined action on the position loop gains. Experimental measurements demonstrate the effectiveness of the proposed approach.
\end{abstract}

Key words: Jerk / Cartesian robot / residual vibration / motion control

\section{Introduction}

\subsection{Problématique générale}

Dans le domaine du positionnement à grande dynamique, l'augmentation des performances en terme de rapidité passe généralement par la diminution des inerties constituant le système. Mais la réduction de masse a pour corollaire l'augmentation du caractère flexible des composants mécaniques. Dès lors, les effets des souplesses mécaniques influencent la réponse dynamique du système

\footnotetext{
a Auteur pour correspondance : barre@lille.ensam.fr
}

d'autant que les accélérations attendues sollicitent encore plus fortement la structure. Une méthode de résolution des problèmes de vibrations mécaniques consisterait à utiliser des matériaux qui absorbent ces dernières. Une telle méthode est toutefois onéreuse et pas toujours totalement satisfaisante [1]. Une autre méthode, investiguée dans cet article, consiste à modifier directement la loi de mouvement imposée au système de façon à tenir compte des effets liés aux flexibilités diverses. En effet, les lois de mouvements classiques à accélération constante par morceau présentent des discontinuités que les asservissements ne peuvent suivre quelles que soient les performances des actionneurs. Ces discontinuités contraignent 


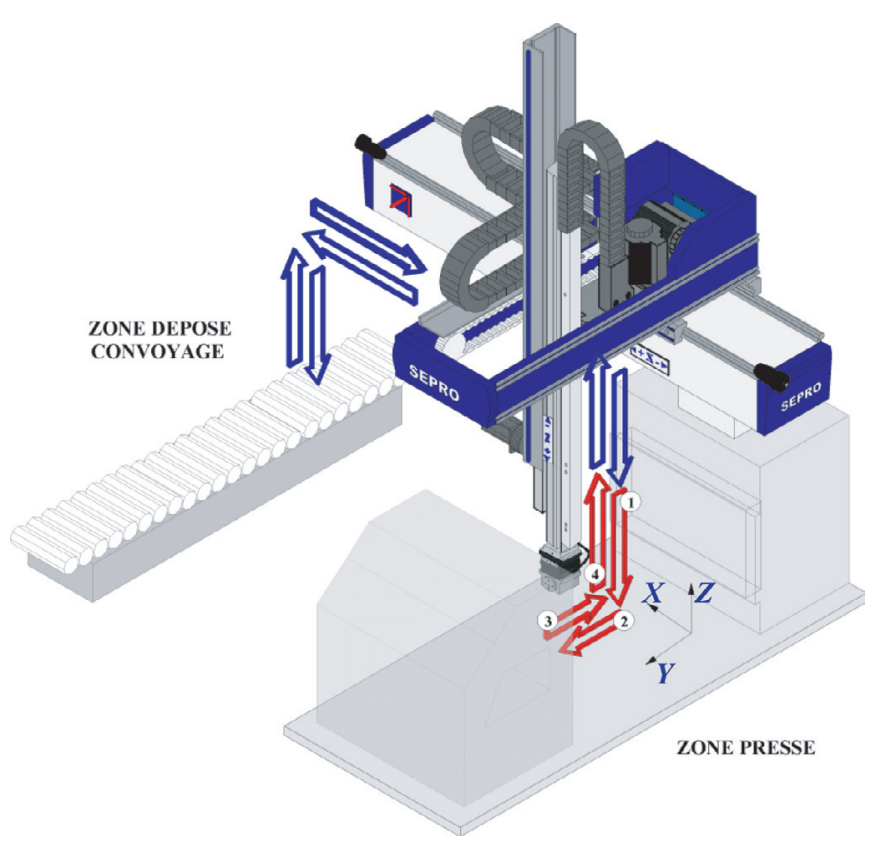

Fig. 1. Cycle type du robot cartésien 3 axes.

la structure lors des phases transitoires et sont responsables, pour une grande part, de la détérioration du comportement dynamique. Dans les commandes numériques de dernière génération, ce phénomène peut être atténué par une action sur le jerk [2]. De façon intuitive, on peut penser que les consignes d'axe seront plus douces donc moins contraignantes pour la mécanique.

L'objectif de cet article consiste à montrer analytiquement l'influence particulière de la valeur du jerk sur le comportement dynamique d'un système de positionnement rapide.

\subsection{Présentation du démonstrateur}

Le démonstrateur utilisé pour cette étude est un robot cartésien 3 axes employé industriellement comme manipulateur pour le déchargement des presses à injecter le plastique. Son rôle consiste à venir prendre une pièce moulée le plus rapidement possible afin de minimiser les temps de presse non productifs puis, à déposer cette pièce au niveau d'un autre poste de travail ou d'un dispositif de convoyage. Lors d'un cycle de déchargement type, représenté à la figure 1 , les sollicitations dynamiques excitent les premiers modes de vibrations de la structure et engendrent des oscillations visibles de l'organe terminal portant la pièce.

Ainsi, l'objectif consiste ici à maîtriser le niveau des amplitudes d'oscillation lors des différentes phases de fonctionnement sans pour autant réduire la productivité de l'ensemble. Le lecteur notera toutefois que les développements qui vont suivre sont généralisables à tout système de positionnement à dynamique élevée; seules les pondérations sur les exigences de rapidité et de précision différeront selon le contexte d'utilisation.

\subsection{Modèle simplifié destiné à l'étude d'influence du jerk}

Une modélisation éléments finis couplée à une analyse modale du robot cartésien 3 axes ont été réalisées en collaboration avec le laboratoire LaRAMA (IFMA ClermontFerrand). Pour l'étude d'influence de la loi de mouvement sur le comportement vibratoire, nous allons naturellement nous intéresser aux premiers modes de vibration; ces derniers contribuent majoritairement au caractère oscillant des axes. Les analyses ont démontré que les premiers modes de flexion du profilé $Z$ dans les directions $X$ et $Y$ (cf. Fig. 2) étaient largement dominants sur la réponse vibratoire de la charge. Pour les deux axes concernés, le modèle générique de commande doit donc tenir compte de la souplesse liée à cette flexion pure.

\section{Modèle de mécanique}

La dynamique d'un système soumis à l'influence d'un mode souple peut se représenter par un couplage souple de deux masses ponctuelles [3]. Ce système, présenté à la figure 3, est composé d'une masse $m_{\mathrm{m}}$ représentant la partie rigide du système en mouvement et, d'une masse $m_{\mathrm{c}}$ représentant la masse de la charge associée au mode souple. Les deux masses sont couplées par une raideur de transmission $K_{\mathrm{t}}$ et par un coefficient d'amortissement visqueux $\mu_{\mathrm{t}}$ représentant respectivement la raideur et l'amortissement interne du mode de déformation longitudinal considéré. L'effort $F$, commandé par le variateur, est transmis par le moteur à la partie mobile de l'axe. Les fonctions de transfert canoniques, déduites des lois de la mécanique entre les vitesses $V_{\mathrm{m}}$ et $V_{\mathrm{c}}$ des deux masses et l'effort moteur $F$, s'expriment dans le domaine continu sous la forme suivante :

$$
\begin{aligned}
& \frac{V_{\mathrm{m}}(s)}{F(s)}=\frac{1+\frac{2 \zeta_{0}}{\omega_{0}} s+\frac{1}{\omega_{0}^{2}} s^{2}}{m_{\mathrm{tot}} s\left(1+\frac{2 \zeta_{0}}{\omega_{0}} s+\frac{1}{\omega_{0}^{2}(1+r)} s^{2}\right)} \\
& \frac{V_{\mathrm{c}}(s)}{V_{\mathrm{m}}(s)}=\frac{1+\frac{2 \zeta_{0}}{\omega_{0}} s}{\left(1+\frac{2 \zeta_{0}}{\omega_{0}} s+\frac{1}{\omega_{0}^{2}} s^{2}\right)}
\end{aligned}
$$

avec :

$$
\begin{aligned}
& m_{\mathrm{tot}}=m_{\mathrm{c}}+m_{\mathrm{m}} ; \quad r=m_{\mathrm{c}} / m_{\mathrm{m}} ; \\
& \omega_{0}=\sqrt{\frac{K_{\mathrm{t}}}{m_{\mathrm{c}}}} ; \quad \zeta_{0}=\frac{\mu_{\mathrm{t}}}{2 \sqrt{K_{\mathrm{t}} \cdot m_{\mathrm{c}}}}
\end{aligned}
$$

\section{Intégration dans la commande}

La structure des asservissements en courant, vitesse et position est réalisée classiquement selon le schéma des boucles en cascades, détaillé notamment dans [4]. La boucle de position contient un simple gain proportionnel; les boucles de vitesse et courant sont régulées par des correcteurs à action proportionnelle/intégrale (cf. Fig. 3). 

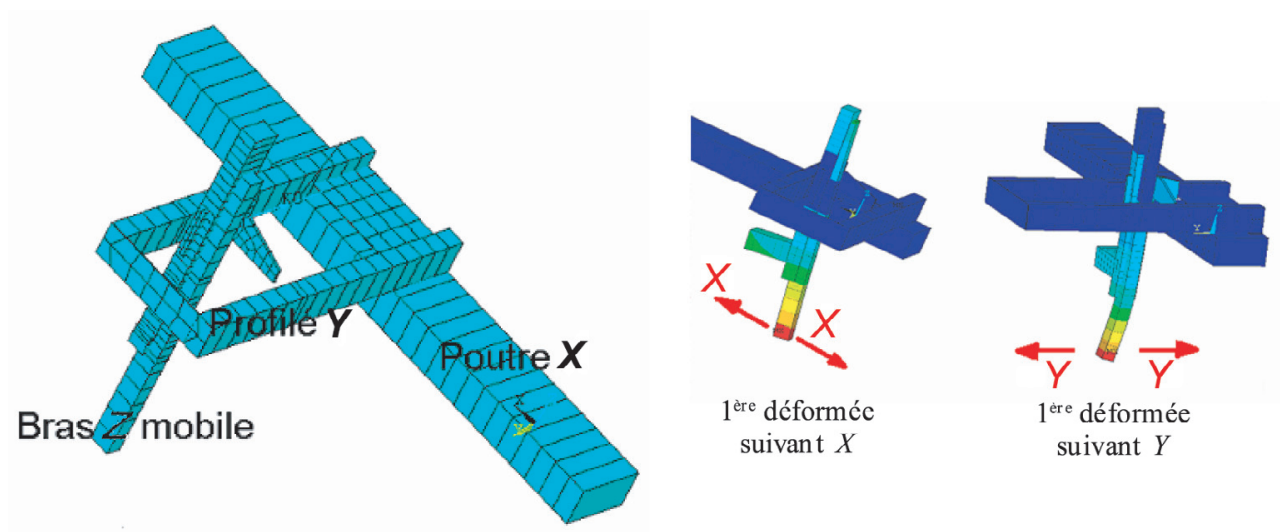

Fig. 2. Modèle éléments finis du robot cartésien et déformées prépondérantes suivant les axes $X$ et $Y$.

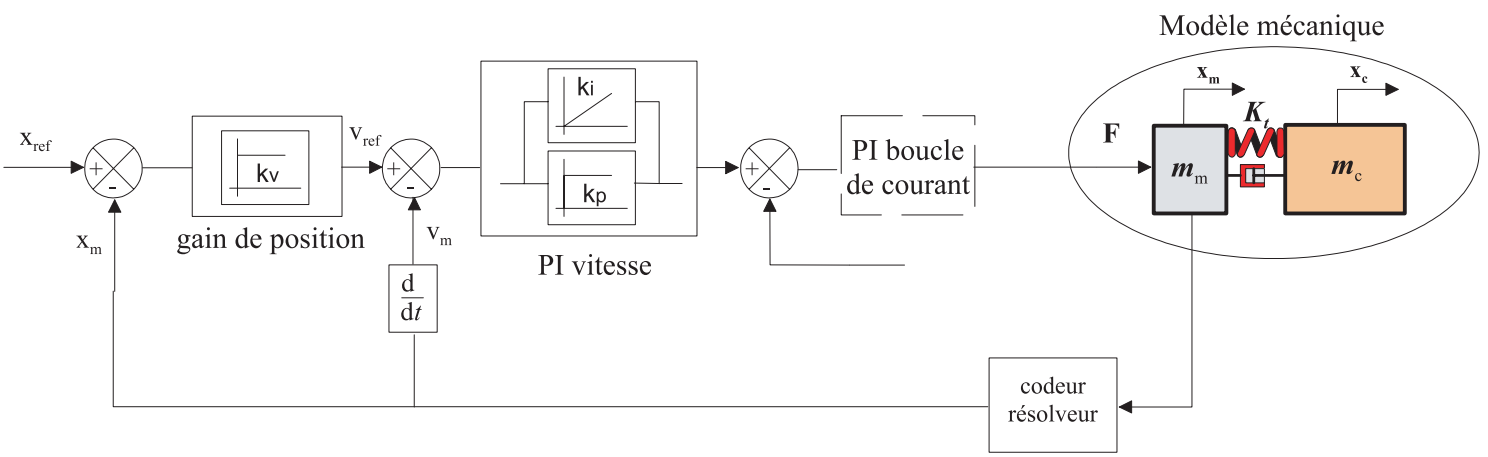

Fig. 3. Architecture de commande classique couplée au modèle d'axe souple.

La mesure est classiquement réalisée au niveau de la masse $m_{\mathrm{m}}$ (mesure indirecte sur le moteur) afin de garantir la stabilité du système. Ainsi, la fonction de transfert $V_{\mathrm{c}} / V_{\mathrm{m}}$ n'est pas directement vue par l'asservissement de vitesse. Si l'on considère à présent l'asservissement de vitesse comme idéal, c'est-à-dire que l'on considère que la vitesse de la masse $m_{\mathrm{m}}$ est égale la vitesse de référence, le transfert global en position entre la charge et la référence de l'axe commandé se résumera à :

$$
\frac{x_{\mathrm{c}}(s)}{x_{\mathrm{ref}}(s)}=\frac{1}{1+\frac{s}{k_{\mathrm{v}}}} \frac{1+\frac{2 \zeta_{0}}{\omega_{0}} s}{\left(1+\frac{2 \zeta_{0}}{\omega_{0}} s+\frac{1}{\omega_{0}^{2}} s^{2}\right)}
$$

en notant $k_{\mathrm{v}}\left[\mathrm{s}^{-1}\right]$ le gain proportionnel de position.

\section{Influence du jerk sur la dynamique du système}

\subsection{Erreur dynamique et loi de mouvement}

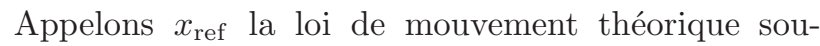
haitée. La présence de discontinuités dans la génération de mouvement aura tendance à exciter les modes souples prépondérants du système et, par conséquent, dégradera la précision dynamique. Le mouvement effectif de la charge noté $x_{\mathrm{c}}$ diffère alors du mouvement idéal. On définit l'erreur dynamique du mouvement :

$$
\varepsilon(t)=x_{\mathrm{c}}(t)-x_{\mathrm{ref}}(t)
$$

On peut dissocier deux types d'erreurs distinctes constituant l'erreur dynamique :

- Des termes apériodiques représentant les écarts liés aux performances en poursuite du système de commande, que l'on notera $\varepsilon_{\text {ap }}(t)$

- Des vibrations liées au comportement oscillant du système que l'on notera $\varepsilon_{\text {vib }}(t)$.

Les lois de mouvement les plus généralement rencontrées sont le profil d'accélération rectangulaire et le profil d'accélération trapézoïdal (jerk limité), présentés à la figure 4. Le premier type de profil permet de réaliser un mouvement sans discontinuité (choc) de vitesse; par contre l'accélération y sera continue par morceaux. Ceci implique des contraintes de chocs d'accélération, appelés également saccades, contribuant fortement à la prépondérance des termes vibratoires dans l'erreur dynamique. Le profil d'accélération à jerk contrôlé offre quant à lui une accélération continue. Cependant, il allonge le temps de cycle d'une durée équivalente à la montée en accélération (notée $T_{\mathrm{j}}$ sur la Fig. 4) pour une même valeur d'accélération maximale. De façon intuitive, plus la valeur de jerk sera faible plus la loi de mouvement sera douce et moins les amplitudes des vibrations induites par cette loi seront importantes. 
(a)

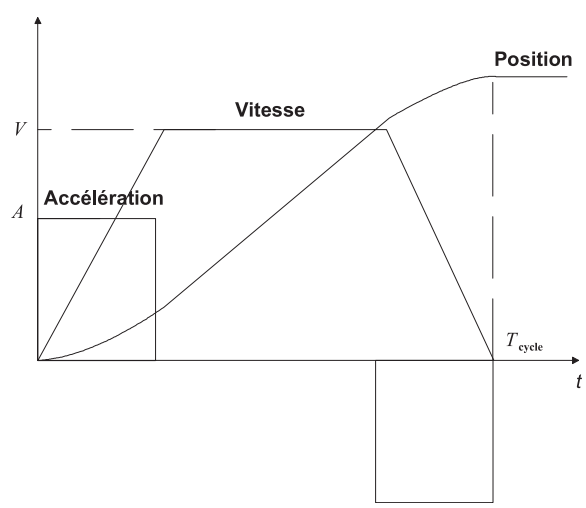

(b)

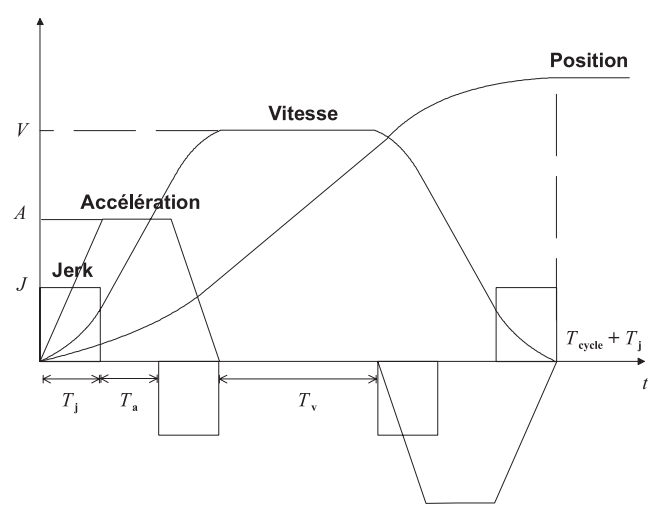

Fig. 4. Lois de mouvement classiques : (a) profil d'accélération rectangulaire, (b) profil d'accélération à jerk contrôlé.

\subsection{Jerk et dynamique vibratoire}

Considérons que la loi de mouvement à jerk contrôlé (cf. Fig. 4b) définie comme suit :

$$
\begin{aligned}
& \ddot{x}_{\mathrm{ref}}(t)= \\
& \begin{cases}J . t & 0 \leq t<T_{\mathrm{j}} \\
A & T_{\mathrm{j}} \leq t<T_{\mathrm{a}}+T_{\mathrm{j}} \\
A-J . t & T_{\mathrm{a}}+T_{\mathrm{j}} \leq t<T_{\mathrm{a}}+2 T_{\mathrm{j}} \\
0 & T_{\mathrm{a}}+2 T_{\mathrm{j}} \leq t<T_{\mathrm{a}}+2 T_{\mathrm{j}}+T_{\mathrm{v}} \\
-J . t & T_{\mathrm{a}}+2 T_{\mathrm{j}}+T_{\mathrm{v}} \leq t<T_{\mathrm{a}}+3 T_{\mathrm{j}}+T_{\mathrm{v}} \\
-A & T_{\mathrm{a}}+3 T_{\mathrm{j}}+T_{\mathrm{v}} \leq t<2 T_{\mathrm{a}}+3 T_{\mathrm{j}}+T_{\mathrm{v}} \\
-A+J . t & 2 T_{\mathrm{a}}+3 T_{\mathrm{j}}+T_{\mathrm{v}} \leq t<2 T_{\mathrm{a}}+4 T_{\mathrm{j}}+T_{\mathrm{v}} \\
0 & 2 T_{\mathrm{a}}+4 T_{\mathrm{j}}+T_{\mathrm{v}} \leq t\end{cases}
\end{aligned}
$$

soit imposée au modèle de commande d'axe souple décrit à l'équation (2). La loi de mouvement utilisée est une succession de rampes et de paliers d'accélération. Ces deux types de phase de mouvement auront une influence différente sur la dynamique vibratoire du système [5].

D'après (2) et (3), le transfert régissant l'évolution de l'erreur dynamique du système souple est donné par :

$$
\frac{\varepsilon(s)}{x_{\mathrm{ref}}(s)}=-\frac{\frac{1}{k_{\mathrm{v}}} s+\left(\frac{1}{\omega_{0}^{2}}+\frac{2 \zeta_{0}}{k_{\mathrm{v}} \omega_{0}}\right) s^{2}+\frac{1}{k_{\mathrm{v}} \omega_{0}^{2}} s^{3}}{\left(1+\frac{s}{k_{\mathrm{v}}}\right)\left(1+\frac{2 \zeta_{0}}{\omega_{0}} s+\frac{1}{\omega_{0}^{2}} s^{2}\right)}
$$

Afin de simplifier les calculs, on supposera dans ce qui suit que l'amortissement est nul.

\section{Rampe d'accélération}

Dans la première phase de mouvement (rampe d'accélération), la transformée inverse du transfert (4) soumis à un échelon de jerk $\left(x_{\text {ref }}=J / s^{4}\right)$ donne l'erreur dynamique suivante :

$$
\varepsilon(t)=\varepsilon_{\text {ap }}(t)+\varepsilon_{\text {vib }}(t)
$$

avec

$$
\begin{aligned}
& \varepsilon_{\text {ap }}(t)= \\
& \quad J\left(\frac{t^{2}}{2 k_{\mathrm{v}}}+\left(t-\frac{1}{k_{\mathrm{v}}}\right) \frac{1-\omega_{0}^{2} / k_{\mathrm{v}}^{2}}{\omega_{0}^{2}}-e^{-t k_{\mathrm{v}}} \frac{\omega_{0}^{3} / k_{\mathrm{v}}^{5}}{1+\omega_{0}^{2} / k_{\mathrm{v}}^{2}}\right)
\end{aligned}
$$

et

$$
\varepsilon_{\mathrm{vib}}(t)=\frac{J}{\omega_{0}^{3}\left(1+\omega_{0}^{2} / k_{\mathrm{v}}^{2}\right)}\left[\frac{\omega_{0}}{k_{\mathrm{v}}} \cos \left(t \omega_{0}\right)-\sin \left(t \omega_{0}\right)\right]
$$

Seul nous intéresse ici le terme oscillant $\varepsilon_{\mathrm{vib}}(t)$. L'amplitude maximale de l'erreur vibratoire dans la rampe sera par conséquent :

$$
\left|\varepsilon_{\mathrm{vib}}\right|_{\max }=\frac{J}{\omega_{0}^{3} \sqrt{1+\omega_{0}^{2} / k_{\mathrm{v}}^{2}}}
$$

\section{Palier d'accélération}

Si, à présent, on calcule l'erreur vibratoire dans le palier d'accélération, c'est-à-dire la réponse du transfert (4) à un échelon d'accélération $\left(x_{\text {ref }}=A / s^{3}\right)$ dont les conditions initiales sont les valeurs de l'erreur dynamique en fin de rampe précédente (pour $t=T_{\mathrm{j}}$ ), on trouve :

$$
\begin{aligned}
& \varepsilon_{\mathrm{vib}}(t)=\frac{A}{T_{\mathrm{j}} \omega_{0}^{3}\left(1+\omega_{0}^{2} / k_{\mathrm{v}}^{2}\right)} \\
& \times\left[\begin{array}{l}
\left(\frac{\omega_{0}}{k_{\mathrm{v}}}\left(\cos \left(T_{\mathrm{j}} \omega_{0}\right)-1\right)-\sin \left(T_{\mathrm{j}} \omega_{0}\right)\right) \cos \left(\bar{t} \omega_{0}\right) \\
-\left(\frac{\omega_{0}}{k_{\mathrm{v}}} \sin \left(T_{\mathrm{j}} \omega_{0}\right)+\cos \left(T_{\mathrm{j}} \omega_{0}\right)-1\right) \sin \left(\bar{t} \omega_{0}\right)
\end{array}\right]
\end{aligned}
$$

$\operatorname{avec} \bar{t}=t-T_{\mathrm{j}}$.

En remarquant que $A=J T_{\mathrm{j}}$, l'amplitude maximale de l'erreur s'écrit :

$$
\left|\varepsilon_{\mathrm{vib}}\right|_{\max }=\frac{2 J}{\omega_{0}^{3} \sqrt{1+\omega_{0}^{2} / k_{\mathrm{v}}^{2}}}\left|\sin \left(\frac{T_{\mathrm{j}} \omega_{0}}{2}\right)\right|
$$

Ou encore en adoptant la notation $\operatorname{sinc}(x)=\sin (x) / x$ :

$$
\left|\varepsilon_{\mathrm{vib}}\right|_{\max }=\frac{A}{\omega_{0}^{2} \sqrt{1+\omega_{0}^{2} / k_{\mathrm{v}}^{2}}}\left|\operatorname{sinc}\left(\frac{T_{\mathrm{j}} \omega_{0}}{2}\right)\right|
$$

L'amplitude de l'erreur vibratoire maximale dans le palier est une fonction sinus cardinal de la durée $T_{\mathrm{j}}$ des phases à jerk constant. La figure 5 présente l'évolution de 


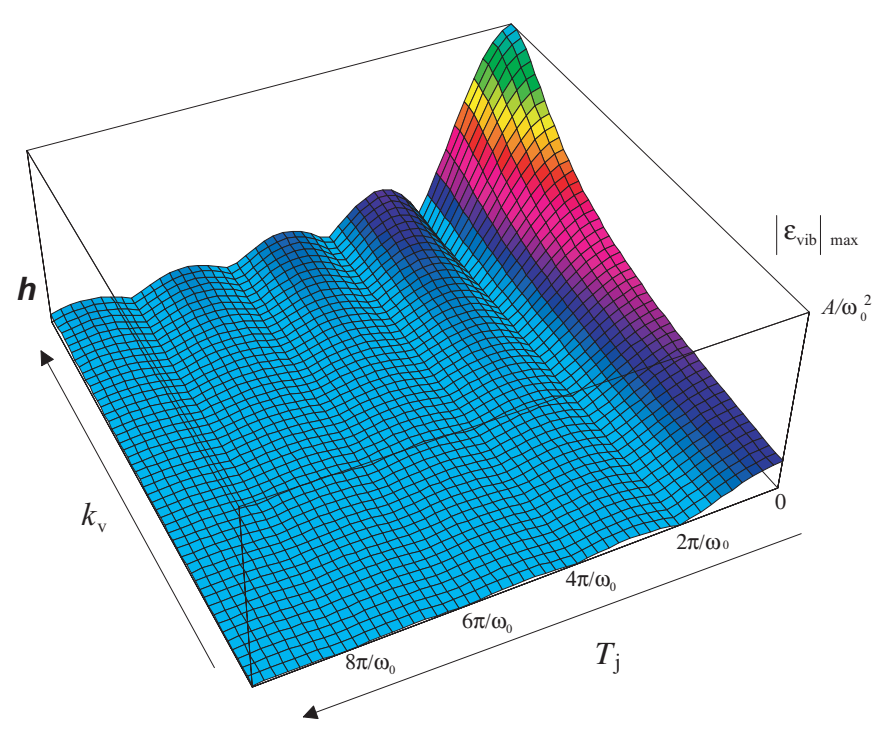

Fig. 5. Évolution des amplitudes vibratoires maximales en fonction de $T_{\mathrm{j}}$ et de $k_{\mathrm{v}}$.

cette erreur en fonction d'une part de la durée des phases à jerk constant et d'autre part du gain de position.

Ainsi, dans le cas particulier où $T_{\mathrm{j}}$ est un multiple entier $n$ de la période propre $T_{\mathrm{j}} \omega_{0}=2 \pi n$, il n'y aura pas de vibration résiduelle dans le palier d'accélération. En dehors de ces points particuliers, on retrouve, de façon triviale, que plus le gain de position est élevé plus l'erreur vibratoire est importante. On notera également que, pour un gain de position donné, on obtient l'erreur vibratoire maximale en faisant tendre $T_{\mathrm{j}}$ vers 0 , ce qui revient à considérer le cas de la loi d'accélération rectangulaire.

\section{Effet cumulatif des oscillations et amortissement}

Le calcul précédent ne reflète que le niveau d'oscillation lors du premier palier d'accélération. En calculant l'erreur vibratoire totale en fin de mouvement (lors de la phase d'arrêt de l'axe), l'amortissement étant supposé nul, on trouverait :

$$
\begin{aligned}
& \left|\varepsilon_{\mathrm{vib}}\right|_{\max }=\frac{4 A}{\omega_{0}^{2} \sqrt{1+\omega_{0}^{2} / k_{\mathrm{v}}^{2}}}\left|\operatorname{sinc}\left(\frac{T_{\mathrm{j}} \omega_{0}}{2}\right)\right| \\
& \quad \times\left|\sin \left(\left(T_{\mathrm{a}}+T_{\mathrm{j}}\right) \omega_{0} / 2\right) \sin \left(\left(T_{\mathrm{a}}+2 T_{\mathrm{j}}+T_{\mathrm{v}}\right) \omega_{0} / 2\right)\right|
\end{aligned}
$$

Lors de la phase d'arrêt, l'erreur vibratoire suit toujours l'évolution décrite par la figure 5; seule l'amplitude de l'erreur diffère. Selon la durée des différentes phases, l'erreur obtenue à l'équation (9) peut être multipliée par 4. Ce résultat est prévisible puisque la loi de mouvement est constituée de 4 paliers d'accélération et que, dans le cas le plus défavorable, les oscillations vont toutes s'ajouter en phase de façon constructive [6]. Toutefois, l'hypothèse retenue sur l'absence d'amortissement est très dure. En effet, l'amortissement du système réduira considérablement l'amplitude de l'erreur. Ainsi, si le mouvement est assez long pour que les oscillations à chaque palier s'amortissent, l'amplitude d'oscillation maximale lors de la phase d'arrêt est donnée par la formule (9). Dans les autres cas, l'amplitude donnée par cette équation est à pondérer (entre 1 et 4 ).

Finalement, les amplitudes d'oscillation lors de la phase d'arrêt diminuent rapidement dès que l'on gère le jerk. La valeur maximum du jerk est actuellement réglée sur chaque axe de façon itérative afin d'obtenir un comportement dynamique acceptable lors d'un mouvement point à point. L'équation (9) montre qu'il est possible de quantifier a priori la valeur maximum du jerk mécanique. En pratique, la durée des phases à jerk constant devra être choisie dans un intervalle de temps compris entre 0 et une période propre du mode prépondérant considéré, ceci en fonction du niveau d'atténuation recherchée (cf. Fig. 5). En effet, au-delà de cette valeur, on ne réalisera aucun gain sur le niveau d'oscillation mais, au contraire, on augmentera inutilement le temps de cycle du système.

\subsection{Jerk et temps de cycle}

Comme vu précédemment, pour atteindre une position donnée, le contrôle du jerk augmente le temps de cycle d'une durée égale à $T_{\mathrm{j}}$ par rapport au profil d'accélération rectangulaire. Le nouveau profil étant moins contraignant pour la mécanique, on peut envisager une augmentation de la valeur d'accélération maximale tolérée. Mais, sans modifier l'accélération maximale, on notera qu'il s'agit ici d'un temps de cycle théorique qui sera dans tous les cas pondéré par le temps de réponse du système, intimement lié à la valeur de son gain de position. Si l'on observe les courbes d'iso-amplitude sur la figure 5 (même teinte), on constate qu'en jouant sur le jerk il est possible d'obtenir le même comportement vibratoire pour tout gain de position. Dans le cas où le $k_{\mathrm{v}}$ est limité par le comportement vibratoire, il apparaît donc que la gestion du jerk permet d'augmenter la valeur de ce gain tout en maîtrisant les oscillations. Il en découle que, si le temps de réponse de l'axe est originellement supérieur à une période propre du mode prépondérant, la gestion du jerk couplée à l'augmentation du $k_{\mathrm{v}}$ permettrait théoriquement de conserver le même temps de cycle, voire même de le réduire. Cependant, si on augmente $k_{\mathrm{v}}$, il faut s'assurer que la fréquence propre du système est convenablement estimée car la sensibilité du niveau vibratoire à l'écart entre $T_{\mathrm{j}}$ et $2 \pi / \omega_{0}$ croît avec $k_{\mathrm{v}}$ (cf. Fig. 5 ).

On notera également que nous nous sommes placés dans le cas d'axes non anticipés en vitesse (cas du robot étudié). Pour les axes anticipés, la réponse vibratoire correspondrait au cas d'une poursuite parfaite, c'est-à-dire au cas $k_{\mathrm{v}} \rightarrow+\infty$ sur la figure 5 . Dans ce dernier cas, la maîtrise du comportement vibratoire par le jerk impliquerait inévitablement une augmentation du temps de cycle.

\section{Validations expérimentales}

Le contrôle du jerk n'est actuellement pas disponible dans la commande industrielle du robot cartésien 

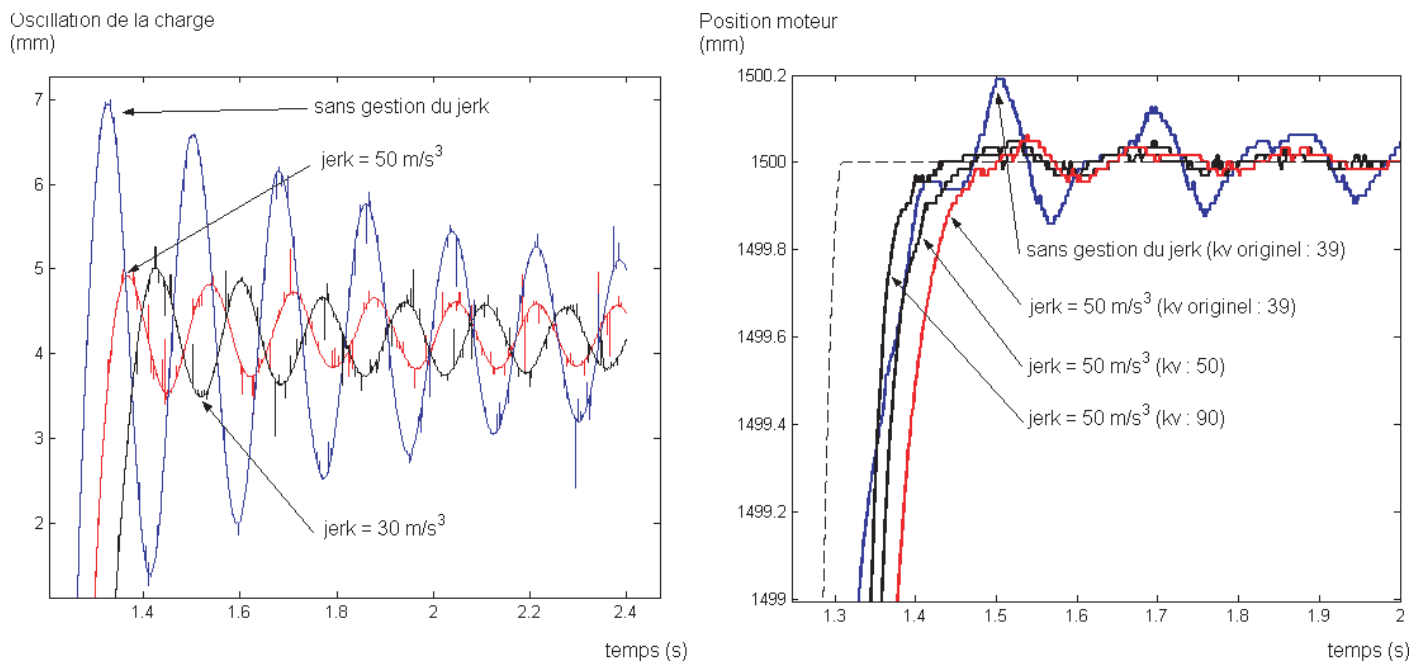

Fig. 6. Oscillations en position suivant $X$ : (a) influence du jerk sur les oscillations de la charge, (b) influence combinée du jerk et du $k_{\mathrm{v}}\left[\mathrm{s}^{-1}\right]$ sur le temps de réponse de l'axe.

PIP 3031. Afin de valider l'influence de ce paramètre, le robot a été équipé d'une carte contrôle temps réel dSPACE 1103. Les mesures disponibles sont issues des codeurs des moteurs d'axe et d'un capteur laser placé au niveau de la charge.

Les résultats présentés ont été obtenus pour un mouvement point à point de $1500 \mathrm{~mm}$ suivant l'axe $X$. L'accélération maximale sur cet axe est fixée à $4,5 \mathrm{~m} . \mathrm{s}^{-2}$ et le gain de position $k_{\mathrm{v}}$ est de $39 \mathrm{~s}^{-1}$. Des mesures par accéléromètres ont permis d'identifier la fréquence des modes prépondérants associés à la poutre $Z$ portant la charge. Suivant l'axe $X$, le premier mode de flexion de la poutre $Z$ est situé à $\omega_{0} / 2 \pi=6,5 \mathrm{~Hz}$. Ainsi, d'après l'équation (10), le niveau d'oscillation théorique maximal (sans amortissement) associé au mouvement sans contrôle du jerk $\left(T_{\mathrm{a}}=0,65 \mathrm{~s}\right)$ est de l'ordre de $3 \mathrm{~mm}$, ce qui est très proche de la valeur mesurée (cf. Fig. 6a).

La durée des phases à jerk constant qui théoriquement annule les oscillations de la charge est donnée par $T_{\mathrm{j}}=2 \pi / \omega_{0} \approx 0,15 \mathrm{~s}$. Pour l'accélération fixée, celles-ci correspondent à un jerk $J$ de l'ordre de $40 \mathrm{~m} . \mathrm{s}^{-3}$. Les figures 6 a et 7 démontrent qu'autour de cette valeur de jerk, les oscillations de la charge sont notablement réduites.

Les figures $6 \mathrm{~b}$ et 7 permettent également de vérifier que, pour la valeur de jerk choisie (dans notre cas $50 \mathrm{~m} . \mathrm{s}^{-3}$ ), il est possible d'augmenter la valeur du gain de position sans dégrader le comportement dynamique. On remarquera qu'il est effectivement possible par ce biais de conserver le temps de cycle originel, voire même de le réduire. Finalement, grâce à la maîtrise du niveau d'oscillation par le jerk, on peut également envisager de réduire notablement le temps de cycle du robot cartésien en augmentant les caractéristiques dynamiques de ses axes.

\section{Conclusions et perspectives}

Le contrôle du jerk pour les systèmes de positionnement à grande dynamique permet effectivement

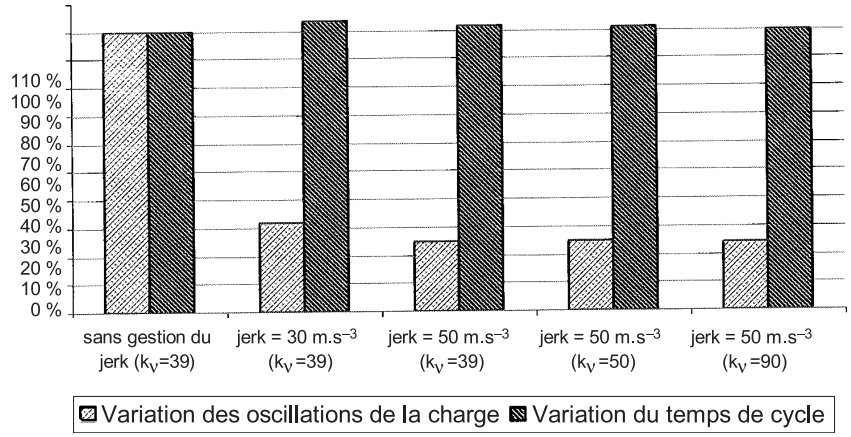

Fig. 7. Graphe d'influence combinée du jerk et du $k_{\mathrm{v}}\left[\mathrm{s}^{-1}\right]$ sur le niveau d'oscillation de la charge et sur le temps de réponse de l'axe comparé au réglage industriel originel (100\%).

d'améliorer le comportement vibratoire des axes. Nous avons démontré que le choix de la valeur maximum du jerk admissible par axe conduisant à un niveau d'oscillation désiré dépend principalement de la position fréquentielle de la souplesse prépondérante de l'axe mais également du gain de position de ce dernier. Les essais expérimentaux ont permis de montrer que l'influence du jerk peut être correctement estimée « a priori » par la formulation théorique développée dans cet article. Nous avons également montré que la gestion du jerk n'implique pas nécessairement l'augmentation du temps de cycle puisqu'elle permet d'augmenter notablement le gain de position dans le cas d'axes non anticipés.

Nous étendons actuellement cette étude afin, d'une part, d'intégrer les effets du pourcentage d'anticipation et, d'autre part, de caractériser les courbes « d'iso temps de cycle $»$ en fonction de l'ensemble des paramètres d'axe. Ce travail constitue le fondement d'une démarche de formalisation de l'effet de la loi de mouvement sur la dynamique des axes. La perspective principale consiste ainsi à investiguer l'influence d'autres lois de mouvement notamment les lois sinusoïdales ou encore polynômiales. 


\section{Références}

[1] R.D. Benning, M.G. Hodgins, G.G. Zipfel, Active control of mechanical vibrations, Bells Labs technical J. (1997)

[2] W. Papiernik, Architecture and design of modern CNC/drive systems, Intelligent motion, May 1996, pp. 271-280

[3] G. Ellis, Control system design guide (2nd edn.), Academic press, Boston, 2000
[4] H. Groß, J. Harmann, G. Wiegârtner, Electrical feed drives in automation, MCD corporate Publishing, SIEMENS, 2001, ISBN 3-89578-148-7

[5] G. Spinnler, Conception des machines. Principes et applications. (Vol. 2 Dynamique), Presses Polytechniques et Universitaires Romandes, Lausanne, 1997

[6] S. Bloch, Gestion des vitesses par la commande numérique en usinage grande vitesse, $1^{\text {re }}$ Assises Machines et Usinage à Grande Vitesse, Senlis, 6-7 mars 2000 